\title{
DISTRIBUCIÓN ESPACIAL Y HÁBITOS DE NIDIFICACIÓN DE NANNOTRIGONA MELLARIA (APIDAE: MELIPONINI) EN UNA LOCALIDAD DE CALI (COLOMBIA)
}

\section{SPATIAL DISTRIBUTION AND NESTING HABITS OF NANNOTRIGONA MELLARIA (APIDAE: MELIPONINI) IN ONE LOCALITY OF CALI (COLOMBIA)}

\section{SERgio MARTÍNEZ LÓPEZ, ${ }^{1, *}$ Edier Alberto SOTO MEDINA, ${ }^{2}$ STEPHANia SANDOVAL ARANGO $^{3}$ Y JOEL TUPAC OTERO OSPINA ${ }^{4}$}

\author{
${ }^{1}$ Universidad Nacional de Colombia sede Palmira, Grupo de investigación en Orquídeas, Ecología y Sistemática \\ Vegetal<Smartinez0101@gmail.com>. \\ ${ }^{2}$ Universidad del Valle <ediersot@gmail.com>. \\ ${ }^{3}$ Universidade de São Paulo, Ribeirão Preto, Brasil, Laboratório de Biologia Comparada e Abelhas (LBCA), \\ Departamento de Biologia, Faculdade de Filosofia, Ciências e Letras de Ribeirão Preto (FFCLRP) \\ <stephania@usp.br>. \\ ${ }^{4}$ Grupo de Investigación en Orquídeas, Universidad Nacional de Colombia sede Palmira, Ecología y Sistemática \\ Vegetal, Instituto de Estudios ambientales (IDEA), Departamento de Ciencias Básicas <jtoteroo@unal.edu.co>. \\ Instituto de Estudios ambientales (IDEA), Departamento de Ciencias Básicas, Universidad Nacional de Colombia \\ sede Palmira, Cra 32 \# 12-00, Chapinero, Palmira, Colombia. \\ * Autor de correspondencia; <Smartinez0101@gmail.com>.
}

Recibido: 13/05/2015; aceptado: 19/04/2017

Editor responsable: Arturo Bonet Ceballos.

Martínez-López, S., Soto-Medina, E. A., Sandoval S. y Otero J. T. (2017). Distribución espacial y hábitos de nidificación de Nannotrigona mellaria (Apidae: Meliponini) en una localidad de Cali (Colombia). Acta Zoológica Mexicana (n.s.) 33(2), 161-168.

RESUMEN. Las abejas Meliponini, son importantes polinizadores y su distribución puede verse afectada de diferentes maneras ante la presencia humana; las urbanizaciones causan cambios al microclima y la estructura y composición de la vegetación, lo que influye sobre sus comunidades. Sin embargo, es mucho lo que se desconoce de su comportamiento en ambientes urbanos. El objetivo del presente estudio fue conocer los hábitos de nidificación de Nannotrigona mellaria en un ambiente urbano. Se realizó un muestreo en el campus Meléndez de la Universidad del Valle, registrando los nidos y el número de colonias por sitio, con lo que se construyó un mapa de distribución usando el programa "DIVA-GIS". Para evaluar la distribución de los nidos se realizó un análisis no espacial de bondad de ajuste a la distribución binomial negativa con una prueba de $\mathrm{Chi}^{2}$. Adicionalmente, se realizó un Análisis Espacial por Índices de Distancia “SADIE”. Se localizaron un total de 242 nidos, de los cuales 130 se encontraron en Pithecellobium dulce (Fabaceae: Faboideae), seguido por los nidos en edificaciones con 91. Los análisis indicaron que los nidos presentan una distribución espacial agregada en el campus de la Universidad del Valle, lo anterior se debe probablemente a diversos factores como la localización y abundancia de recursos, los espacios aprovechables para la nidificación (troncos huecos o grietas en edificaciones) y las limitaciones en cuanto a la capacidad máxima de vuelo debido a su tamaño. A pesar de la preferencia por nidificar en $P$. dulce, las edificaciones son im-
Martínez-López, S., Soto-Medina, E. A., Sandoval S., \& Otero J. T. (2017). Spatial distribution and nesting habits of Nannotrigona mellaria (Apidae: Meliponini) in one locality of Cali (Colombia). Acta Zoológica Mexicana (n.s.) 33(2), 161-168.

ABSTRACT. Meliponini bees are important pollinators in natural habitats and their distribution may be affected differently by humans, the buildings causing changes in micro-climate and vegetation structure and composition, which may affects stingless bee communities. However, there is much to know about their behavior in these conditions. The aim of this study was to describe nesting habits of Nannotrigona mellaria at an urban environment. Sampling was conducted at the Universidad del Valle, Melendez campus, the nest location and number of colonies per site, were recorded and mapped using the program DIVA-GIS. The nest distribution was analyzed with a non-spatial analysis performed by adjusting the data to the negative binomial distribution with a $\mathrm{Chi}^{2}$ test. Additionally, Spatial Analysis by Distance Indices (SADIE) was performed. We found 242 nests, of which 130 were on Pithecellobium dulce (Fabaceae: Faboideae), and 91 where found in buildings. Analyses suggested that Nannotrigona mellaria nests have an aggregated distribution at the Universidad del Valle, this probably due to factors such as the location and abundance of resources, usable spaces for nesting and limitations as to the maximum flight because the size of the bees. In addition, $P$. dulce was mainly used to establish colonies, however, it is clear that buildings are an important space resource for this species and provide the necessary conditions for the establishment of colonies in an urban habitat. 
portantes para esta especie y brindan condiciones necesarias para el establecimiento de las colonias en ambientes urbanos.

Palabras clave: abejas eusociales, agregación, ecología urbana, Pithecellobium dulce, "SADIE".

\section{INTRODUCCIÓN}

Las abejas sociales son importantes polinizadores y visitantes florales en las comunidades vegetales (Free, 1993); entre éstas, las meliponinas o abejas sin aguijón (Apidae: Meliponini), son consideradas entre los principales polinizadores en el neotrópico, debido a que han establecido estrechos lazos co-evolutivos de interdependencia simbiótica con la flora nativa (Monge, 2001) y que existen cientos de especies distribuidas en todas las zonas tropicales del mundo, siendo el grupo de abejas con mayor diversificación (Michener, 2007).

A pesar de su importancia, se desconoce la riqueza y diversidad de abejas sin aguijón en muchas regiones; además su biología y ecología han sido poco investigadas (Jarau \& Barth, 2008). Se sabe que los meliponinos, en particular en el neotrópico, muestran una gran diversidad de estilos de vida y ecología, pero son pocas las investigaciones realizadas, y la información existente de los hábitos de nidificación es limitada (Fernández et al., 2010). Conocer los hábitos de nidificación en meliponinos es importante, ya que diferentes aspectos de la arquitectura y localización de las colonias, revelan aspectos de la historia natural de estas abejas, en relación a su respuesta frente a las variaciones y exigencias del medio externo (Vergara et al., 1986).

La mayoría de las especies de meliponinos en la región central de Colombia, utilizan árboles vivos para establecer sus nidos, y el número de sustratos que utilizan puede variar entre las especies (Vergara et al., 1986). Por ejemplo, especies como Paratrigona isopterophila (Schwarz, 1934), Scaptotrigona limae (Brèthes, 1920) y Trigona fulviventris Guérin 1844, nidifican en un solo tipo sustrato, mientras otras como las del género Nannotrigona pueden construirlos en diferentes tipos, tales como cortezas de árboles, grietas de edificaciones e incluso en el suelo (Vergara et al., 1986).

La especie Nannotrigona testaceicornis (Lepeletier, 1836) es la más estudiada dentro del género; anida en cavidades de árboles o cavidades en estructuras hechas por el hombre (Nogueira-Neto, 1997), sin embargo, la información sobre la preferencia por alguna especie de árbol para nidificar aún es desconocida. En Colombia, estos estudios se han realizado para algunas especies de
Key words: aggregation, eusocial bees, Pithecellobium dulce, SADIE, urban ecology.

meliponinos entre los que se encuentra Nannotrigona melanocera (Schwarz, 1938), en la zona centro occidental de Colombia (Mantilla \& Nates-Parra, 2006; Nates-Parra et al., 2008). No obstante para este grupo de abejas en general, las preferencias de nidificación han sido poco estudiadas en el país.

En Nannotrigona mellaria (Smith, 1862), una especie muy abundante en la ciudad de Cali (Colombia), se desconocen muchos de los aspectos de su ecología e historia natural, tales como hábitos de nidificación y preferencia de sustratos. Se sabe que estas especies construyen sus nidos principalmente en árboles vivos, aunque a veces también en el suelo y edificaciones (Roubik, 1992); pero al igual que con la mayoría de las Meliponini, no hay información sobre la identidad de las especies de árboles en los que nidifica, el tipo de material utilizado y la altura a la que construye los nidos. El objetivo de este trabajo fue determinar los hábitos de nidificación de $N$. mellaria, incluyendo información de las especies de árboles preferidas por esta especie, la altura a la que construyen sus nidos y la distribución espacial de sus colonias en el campus de la Universidad del Valle, Cali, Colombia.

\section{MATERIALES Y MÉTODO}

Área de estudio. El estudio se realizó en el campus Meléndez de la Universidad del Valle, localizado al sur de la ciudad de Cali, perteneciente al departamento del Valle del Cauca, Colombia. El campus se encuentra en las coordenadas $3^{\circ} 22^{\prime} \mathrm{N} 76^{\circ} 31^{\prime} \mathrm{O}$, en un área aproximada de 100 ha, a una altura de $995 \mathrm{~m}$ snm, con una temperatura promedio de $24{ }^{\circ} \mathrm{C}$ y humedad relativa de $75 \%$ (HerreraHurtado, 2009), por lo cual se cataloga como una zona de vida de bosque seco tropical (Bs-T) según la clasificación de Holdridge (1967).

Método de muestreo. Mediante un pre-muestreo en el campus de la Universidad del Valle se recolectaron especímenes de varias especies de abejas sin aguijón entre junio y julio de 2012, que posteriormente fueron identificadas con la ayuda del Laboratorio de Investigación en Abejas de la Universidad Nacional de Colombia Sede Bogotá (LABUN) y fueron depositados en su colección entomológica. Para este trabajo se utilizó la especie que 
presentaba un mayor número de colonias, Nannotrigona mellaria; estas abejas presentan una entrada tubular erecta, compuesta de cera y con una longitud variable de hasta $10 \mathrm{~cm}$ aproximadamente (Fig. 1).

Un grupo de tres personas realizó la búsqueda de nidos de esta especie durante dos semanas en el área total del campus, registrando los que fueran visibles desde el nivel del suelo hasta una altura aproximada de $10 \mathrm{~m}$. En todos los árboles y edificaciones, se anotó la altura a la que estaba la entrada además del tipo y características físicas del sustrato en el que se encontraba (especie de árbol, nido en corteza o en tronco hueco, tipo de edificación). Las coordenadas de cada nido se obtuvieron con ayuda del programa Google Earth (disponible en https://www. google.es/intl/es_es/earth/learn/). En el caso de encontrar más de un nido en un sustrato determinado, se registró el número total de nidos en la misma coordenada.
Análisis de datos. Mediante el uso del programa "DIVA-GIS” (Hijmans et al., 2001), se generó una grilla de cuadrantes de 0.0025 X 0.0025, sobre la Universidad del Valle, y se ubicaron los sitios en los cuales se encontraron colonias de $N$. mellaría. Con estos datos se realizó un Análisis Espacial por Índices de Distancia "SADIE”, con 5967 permutaciones y 17975 de semilla (Perry \& Hewitt, 1991). Adicionalmente, se realizó un análisis no espacial con el fin de determinar si los datos se ajustan a una distribución geométrica (Binomial negativa) por medio de una prueba de $\mathrm{Chi}^{2}$ y de esta manera determinar si hay agrupamiento de nidos en cuadrantes específicos. Por último, se comparó mediante el uso de histogramas la altura a la que nidifican las abejas usando los datos de los dos tipos de sustrato más utilizados por ellas. Estos análisis se realizaron mediante el uso del programa informático STATISTICA 8 de Statsoft.

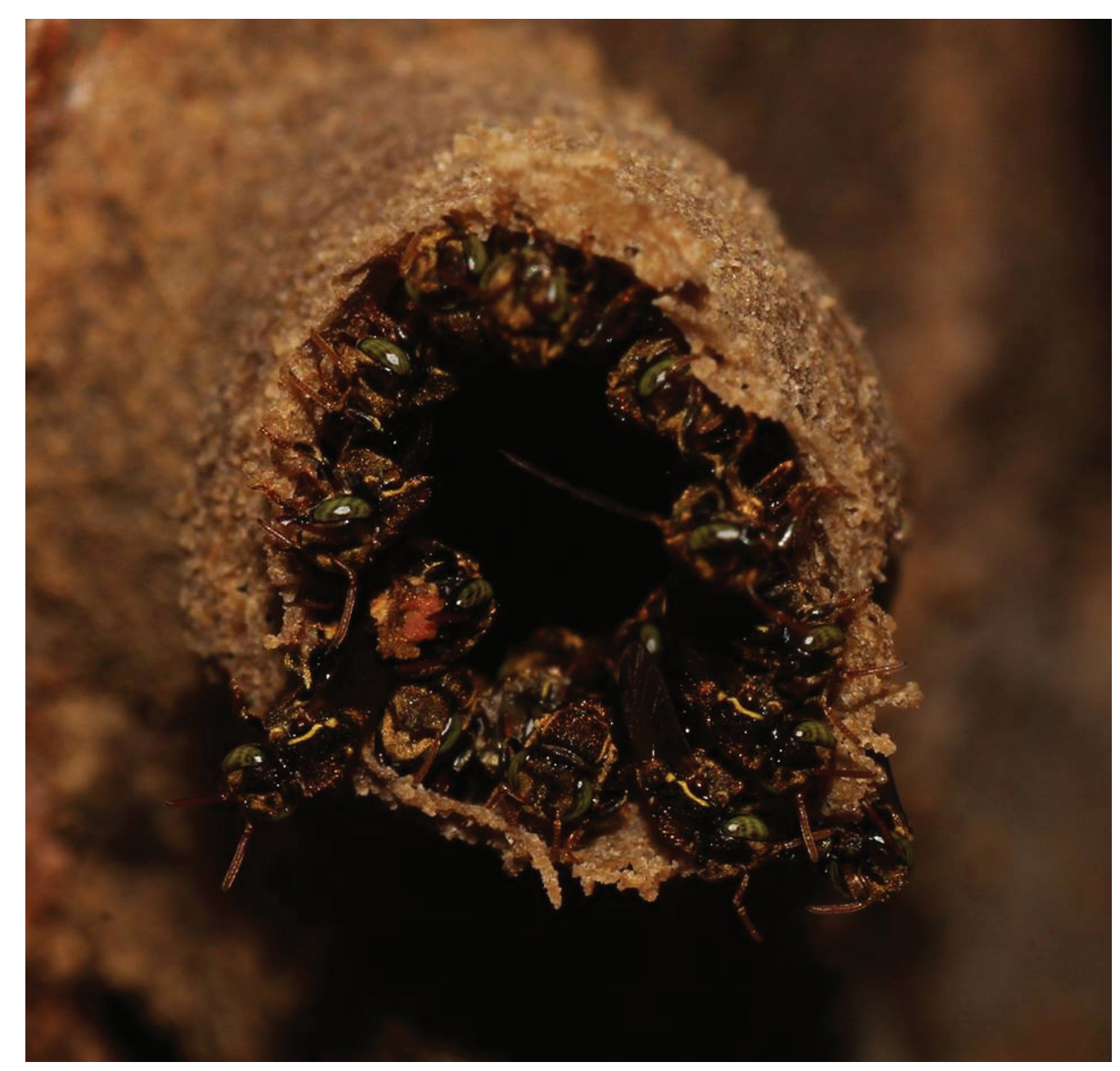

Figura 1. Entrada al nido de Nannotrigona mellaria. 


\section{RESULTADOS}

En total se localizaron 242 nidos de $N$. mellaria, ubicados en siete especies diferentes de árboles y también en edificaciones, siendo Pithecellobium dulce (Roxb.) Bent la especie preferida para nidificar; en ésta se encontraron 130 nidos, correspondiente al 57.72\%, seguido en abundancia por las construcciones humanas, en las que se encontraron 91 nidos en total, es decir 37.61\% (Cuadro 1).

Las entradas de los nidos se encontraron a diferentes alturas, desde el nivel del suelo hasta aproximadamente $8.5 \mathrm{~m}$, la mayoría se ubicaron en el intervalo entre $0-1.5$ $\mathrm{m}$. Se registró que $N$. mellaria nidifica en $P$. dulce principalmente a alturas entre $1-1.5 \mathrm{~m}$ (altura máxima de 3.03 m) (Fig. 2), en comparación, los nidos en edificaciones se encuentran con mayor frecuencia entre los $0-1 \mathrm{~m}$. Sin embargo, en este sustrato se encontraron hasta los 8.5 $\mathrm{m}$, una altura máxima mucho mayor que la reportada en P. dulce (Fig. 3). Una de las características físicas más notables de las edificaciones es que presentaban muchas cavidades en las paredes de ladrillo, grietas en el cemento de paredes y escaleras, además de tuberías.

En cuanto a la distribución de los nidos, se encontró que en esta especie existe una tendencia a nidificar de manera agregada, pues fue común encontrar más de una
Cuadro 1. Número de colonias de Nannotrigona mellaria por sitio de nidificación en la Universidad del Valle, Cali (Colombia).

\begin{tabular}{lccc}
\hline \multicolumn{1}{c}{ Sitio de nidificación } & $\mathrm{n}$ & porcentaje & $\begin{array}{c}\overline{\mathrm{X}} \text { nidos/sitio } \\
\text { de nidificación }\end{array}$ \\
\hline $\begin{array}{l}\text { Pithecellobium dulce } \\
\text { (Roxb.) Benth. }\end{array}$ & 130 & 53,72 & 2 \\
Edificaciones & 91 & 37,6 & 2,46 \\
$\begin{array}{l}\text { Guazuma ulmifolia Lam. } \\
\text { Sapindus saponaria L. }\end{array}$ & 5 & 2,07 & 1 \\
$\begin{array}{l}\text { Ficus benjamina L. } \\
\text { Spathodea campanulata P. }\end{array}$ & 5 & 2,07 & 1,67 \\
$\begin{array}{l}\text { Beauv } \\
\text { Talipariti tiliaceum (L.) }\end{array}$ & 1 & 2,07 & 5 \\
$\begin{array}{l}\text { Fryxell } \\
\text { Clitoria fairchildiana R. A. }\end{array}$ & 1 & 0,41 & 1,67 \\
Howard & & & 1 \\
\hline
\end{tabular}

colonia por árbol o por construcción humana; además, en algunos casos se observaron nidos en árboles y estructuras aledaños. El ajuste con la distribución binomial negativa reportó un valor $\mathrm{Chi}^{2}=5.199(\mathrm{P}=0.158)$. Este resultado indica que en los cuadrantes se presenta un mayor número de espacios sin colonias de los que cabría esperar si se distribuyeran de manera uniforme en el área (Fig. 4).

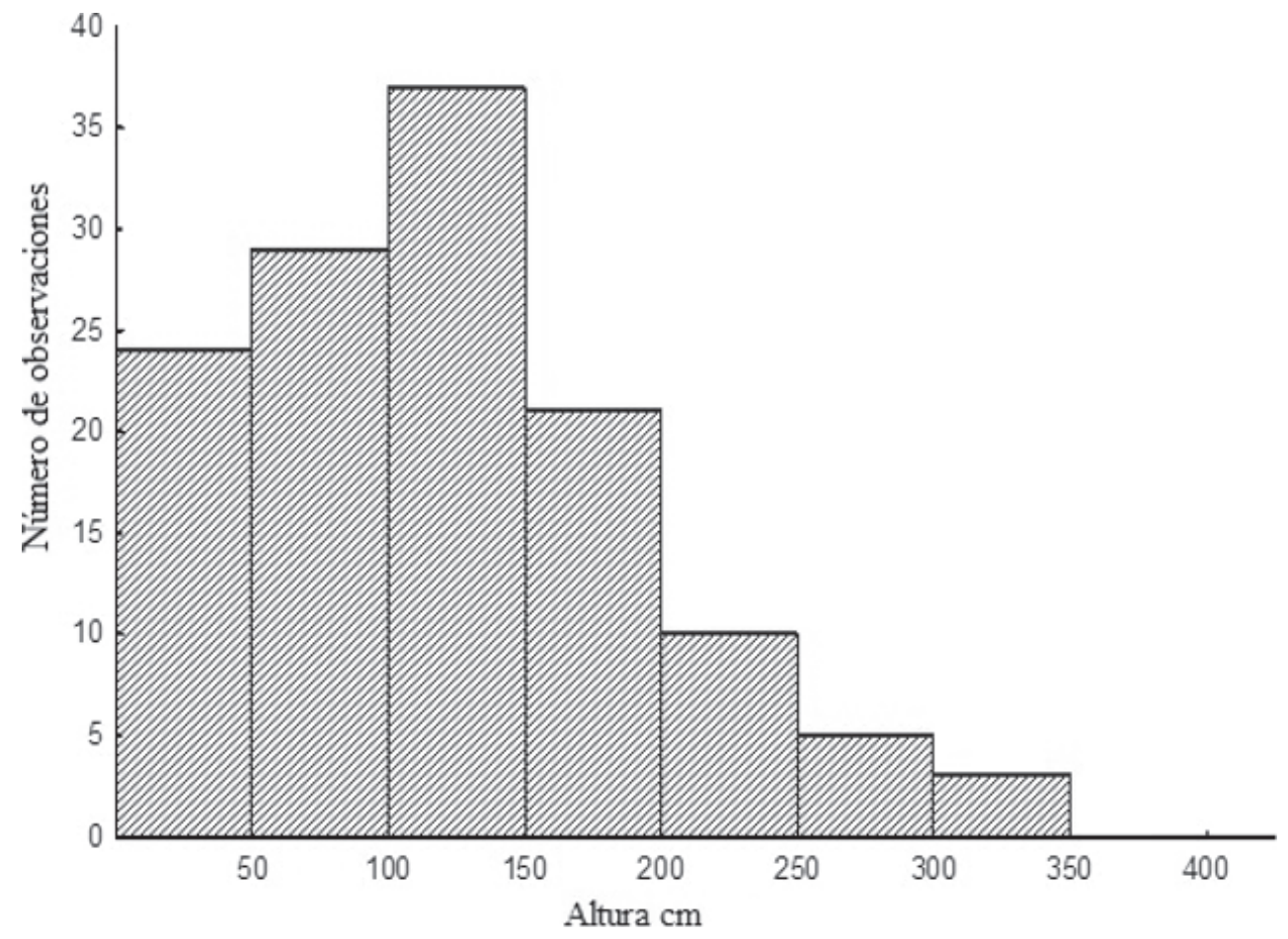

Figura 2. Altura de nidificación de Nannotrigona mellaria en Pithecellobium dulce de la Universidad del Valle, Cali (Colombia), julio 2012. 


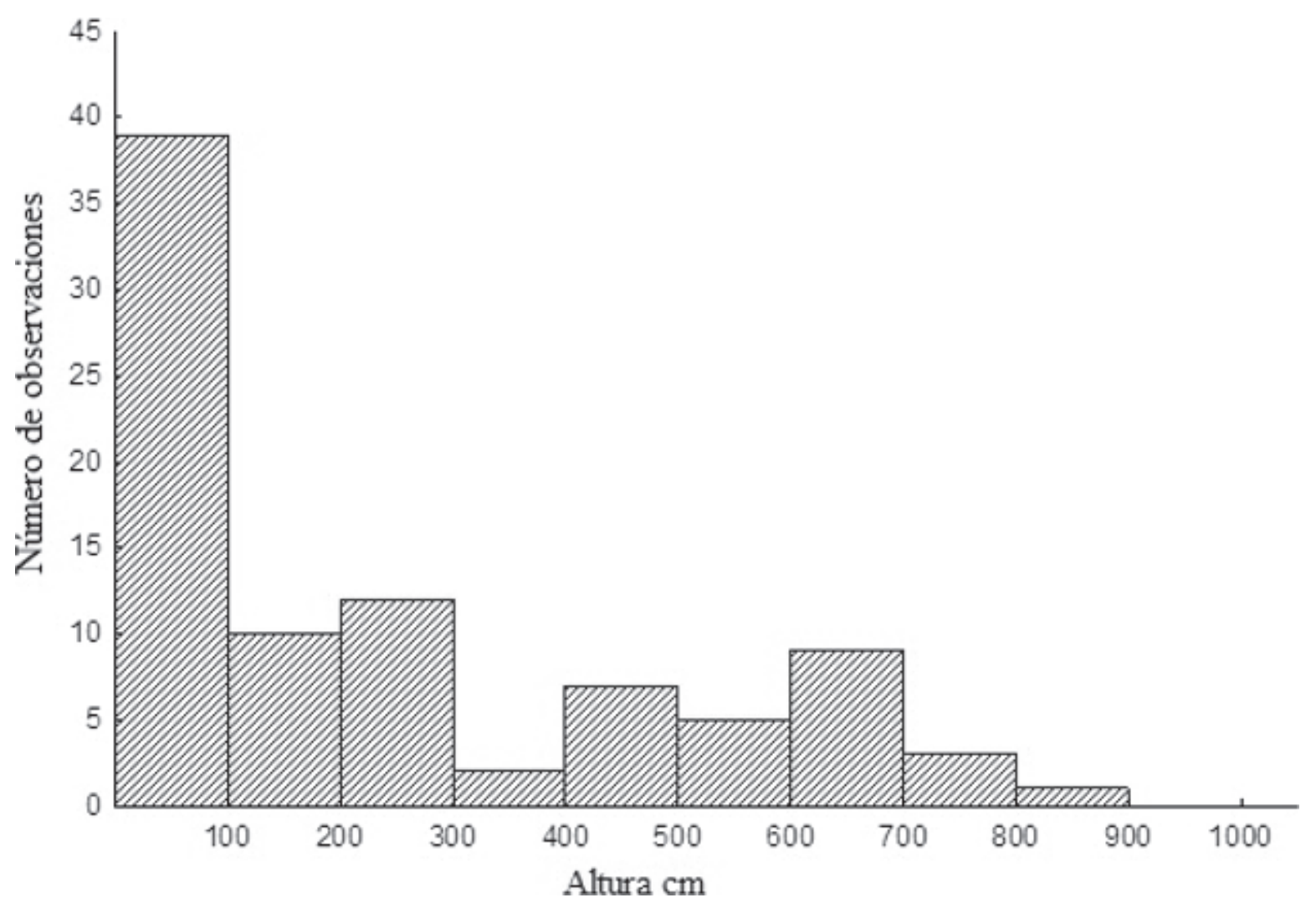

Figura 3. Altura de nidificación de Nannotrigona mellaria en edificaciones de la Universidad del Valle, Cali (Colombia), julio de 2012.

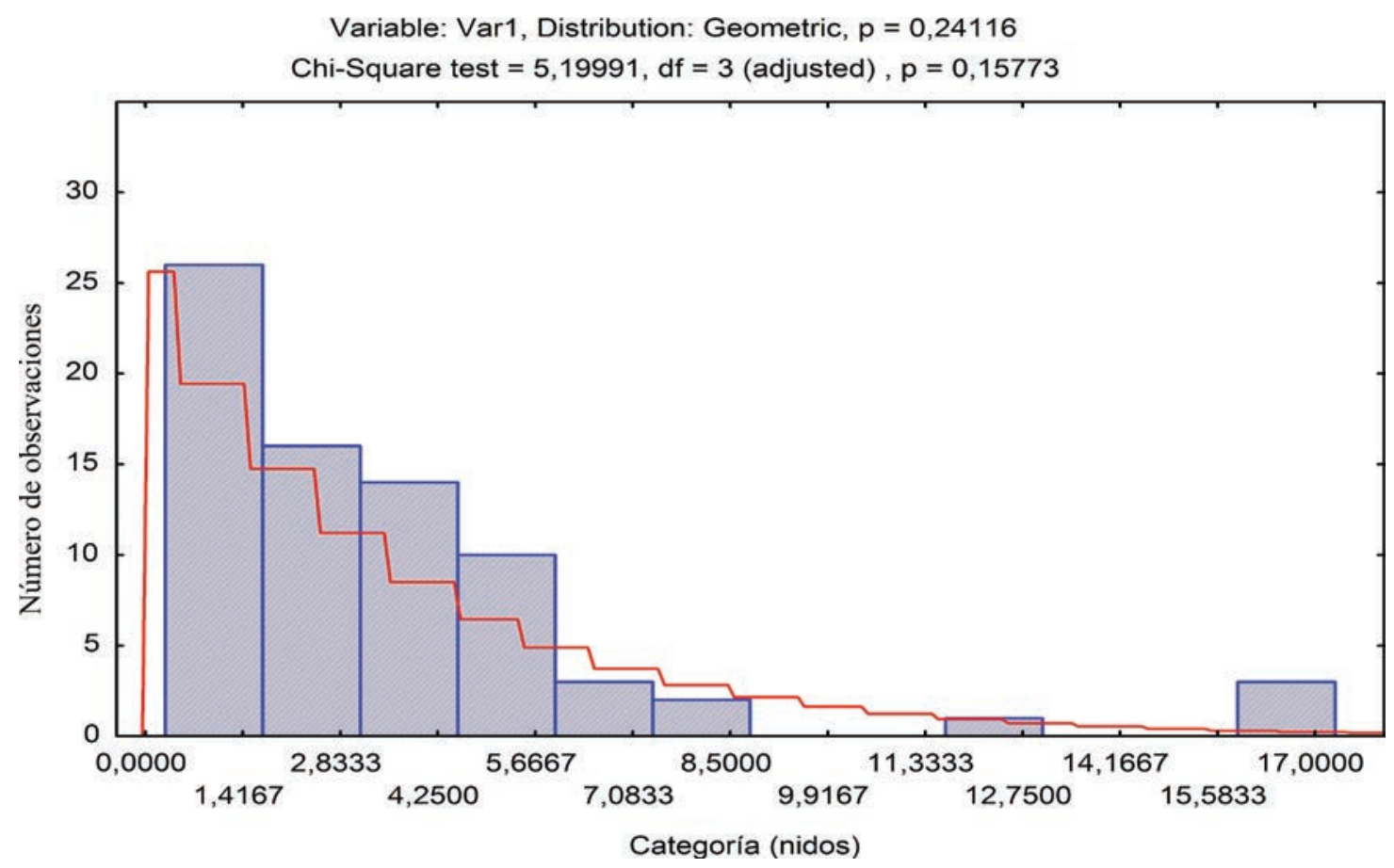

Figura 4. Prueba de $\mathrm{Chi}^{2}$ de ajuste a la distribución binomial negativa. 
Se reportó un número máximo de 16 colonias por cuadrante, lo que se presentó en sólo tres de estos (Fig. 5). El resultado del análisis “SADIE” presentó los valores: $\mathrm{D}=$ 0.374 , un índice de agregación $I a=1.485 ; \mathrm{Pa}=0.016$. El índice de agregación Ia describe el patrón espacial de los datos: comúnmente el patrón espacial es agregado cuando $I a>1$, aleatorio si $I a=1$ y regular si $I a<1$.

\section{DISCUSIÓN}

El recurso más utilizado para la nidificación de abejas sin aguijón son las cavidades arbóreas (Roubik 1979, 1983, 1989; Slaa 2003). En el caso de N. mellaria de los 4250 árboles y las 182 especies de árboles que se encuentran en el campus de la Universidad del Valle (Herrera-Hurtado, 2009), las cavidades en los árboles de $P$. dulce fueron el sustrato más utilizado con alrededor del $53 \%$ de los reportes en el muestreo. Estos árboles tienen algunas características morfológicas como una disposición "enredada" de las ramas, que forma nudos en su base, con corteza ligeramente fisurada, además de ser fuente de néctares y polen (Crane et al., 1984; Vásquez et al., 1999) por lo que puede ser atractivo para el establecimiento de nuevas colonias.

Sin embargo, es posible que uno de los factores más importante para el establecimiento de nuevas colonias en esta especie sea la disponibilidad de cavidades sin importar el tipo de sustrato. Algunas meliponinas exploran y prefieren lugares con sitios de anidamiento disponibles, específicamente huecos, independientemente de si se encuentran en condiciones naturales o en construcciones urbanas, incluso en cementerios (Nates-Parra et al., 2006; Fierro et al., 2012); además de P. dulce, las construccio-
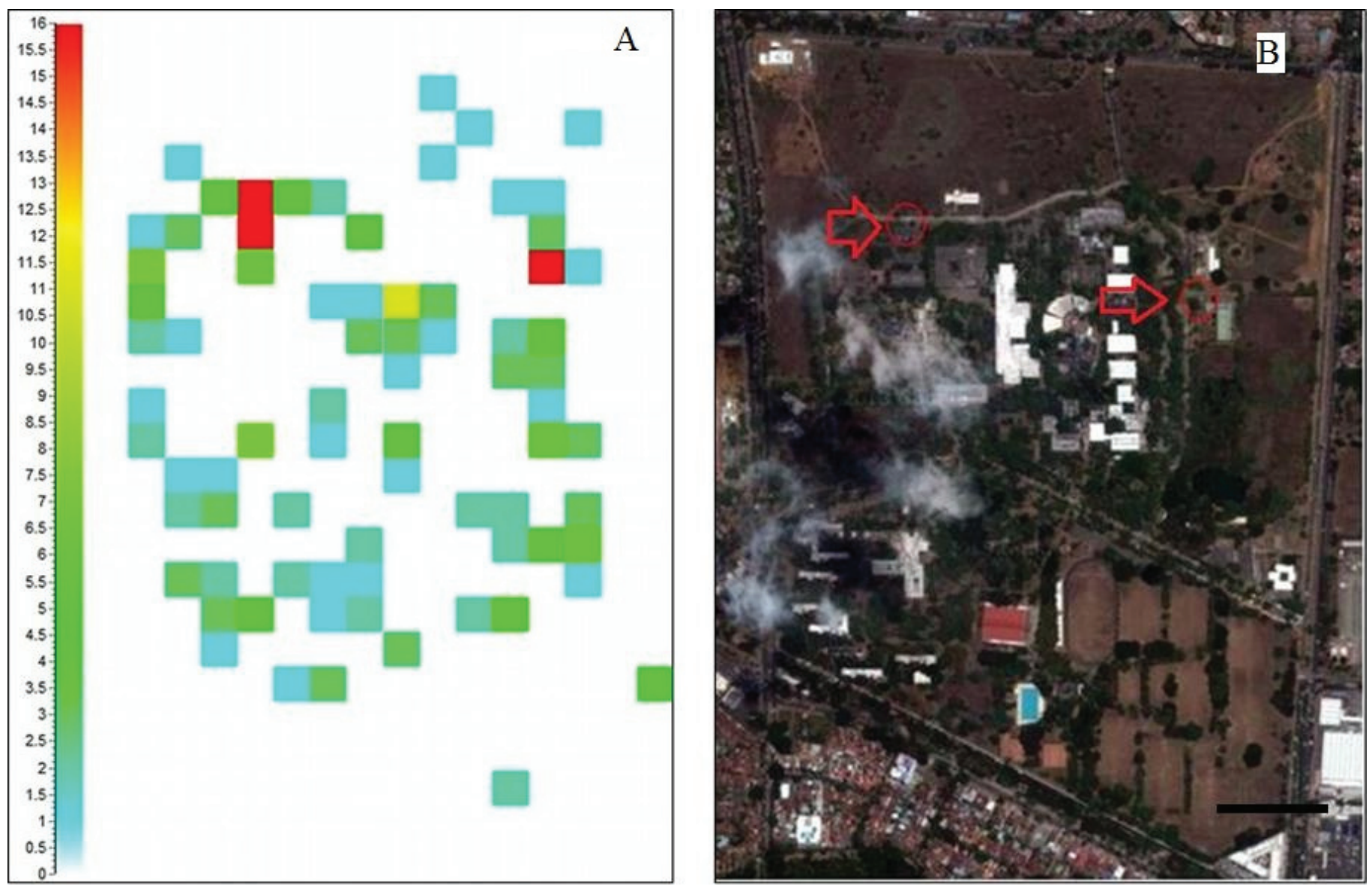

Figura 5. Representación por cuadrantes en escala de 0 a 16 nidos. A) Número de nidos por cuadrantes en la Universidad del Valle, Cali (Colombia), Julio de 2012. B): Foto satelital del campus de la Universidad del Valle Meléndez ubicando los sitios donde los cuadrantes presentaron un mayor número de nidos. Escala 200 m (imagen tomada de Google Earth ()). 
nes presentaban algunas características en las paredes de ladrillo, cemento, escaleras y tuberías donde hay cavidades que facilitan la instauración de los nidos.

Así mismo, la altura a la que se encuentra la entrada de las colonias puede estar limitada por la disponibilidad de espacios huecos donde establecer las colonias. Como se mencionó anteriormente, $P$. dulce presenta algunas características morfológicas que facilitan el establecimiento de nidos, sin embargo, la mayoría de las cavidades en esta especie se encuentran a baja altura, donde el DAP es mayor. Esta limitante no se aplica en el caso de las construcciones, ya que en estas pueden hallarse cavidades a diferentes alturas y es posible que esta sea la razón por la cual las entradas en estas, se encontraron en sitios significativamente más altos, llegando hasta $8.5 \mathrm{~m}$ de altura.

Respecto a los análisis estadísticos utilizados, el análisis no espacial presentó valores de $\mathrm{Chi}^{2}$ que indican que no existen diferencias estadísticas significativas en cuanto a los datos de abundancia de nidos por parcela, al ajustarlos con la distribución binomial negativa. Sin embargo, el análisis "SADIE" tiene en cuenta la localización espacial de los nidos muestreados y resulta más intuitivo y biológico que los índices no espaciales (Perry, 1995). De acuerdo a estos resultados, existe una tendencia a la agregación de las colonias de $N$. mellaria en el área de estudio.

Adicionalmente, existen tres cuadrantes dentro del área de estudio en las que se reportaron un total de 16 nidos (Fig. 5), un número mayor de colonias de las que se presenta en los demás. En estos cuadrantes, las condiciones ambientales para el establecimiento de colonias (cantidad de árboles y/o edificaciones) no difieren de aquellos en los que se reportaron un menor número de colonias, pero si varía respecto a los claros donde no se encontró ninguna. En general, los sitios donde se reporta la presencia de nidos cuentan con coberturas arbóreas y/o edificaciones muy cercanas, mientras que los claros son generalmente áreas abiertas como pastizales.

Es importante mencionar que entre las abejas meliponinas, el mecanismo para la dispersión de los nidos parece estar relacionado con la interacción agresiva directa (una especie excluye a otra de manera agresiva), bien sea por sitios de anidamiento o por los recursos en la zona (Johnson \& Hubbell, 1974); sin embargo, algunas especies de abejas no agresivas suelen establecer sus nidos de manera agregada o aleatoria excepto cuando la competencia por la comida es muy alta (Hubbell \& Johnson, 1977; Slaa 2006). Entre los géneros que más comúnmente se ha reportado este comportamiento, están las abejas de los géneros Scaptotrigona, Partamona, Nannotrigona y Te- tragonisca (Michener, 1946; Hubbell \& Johnson, 1977; Roubik, 1983).

En algunos nidos se observó un comportamiento agresivo entre $N$. mellaria y las especies Apis mellifera L. y Scaptotrigona cf. limae (Brèthes, 1920) y a nivel intra específico en pocas ocasiones, por lo que sería interesante realizar investigaciones en las que se pueda determinar cómo son las interacciones entre las abejas eusociales dentro del campus universitario y si existen patrones de nidificación entre las especies allí presentes.

Contrastando con las abejas Apini, las Meliponini inician la construcción de los nidos mediante visitas constantes, cargando materiales de construcción y alimentos desde la colonia fundadora. Luego una joven reina se desplaza al nuevo sitio elegido y el nido se independiza de la colonia madre gradualmente en un periodo de semanas o meses, así pues, la dispersión a largas distancias por individuos reproductivos o enjambrazones resulta imposible (Nogueira-Neto, 1997; Michener, 2007).

Debido al tamaño del área muestreada (100 ha) y a que no existen barreras geográficas muy marcadas, es probable que exista solo una población dentro del campus de la Universidad del Valle. Sin embargo, al ser abejas de pequeño tamaño, existen limitaciones en cuanto a la capacidad máxima de vuelo (Araújo et al., 2004); ésto sumado a factores como la presencia de espacios aprovechables para nidificación, localización, abundancia y disponibilidad de recursos florales, hábitos en la formación de nuevas colonias entre otros, puede limitar de manera directa la capacidad de dispersión de las poblaciones en esta especie.

\section{CONCLUSIONES}

Las abejas de la especie $N$. mellaria tienen una preferencia por los árboles de $P$. dulce al momento de establecer sus nidos. También, se reporta una importante presencia de nidos en agujeros de construcciones humanas. Así pues, esta especie tolera la presencia humana y puede llegar a hacer uso de las construcciones brindando servicios ecosistémicos de polinización a una parte importante de la flora urbana en la ciudad de Cali. De igual manera, se determinó que la altura a la que nidifican es menor en árboles de $P$. dulce que en edificaciones, posiblemente debido a la limitación en los sitios disponibles para el establecimiento de colonias. Estudios posteriores podrían evaluar el uso de sustratos por esta especie en condiciones naturales y esclarecer las variables que determinan el es- 
tablecimiento de una colonia. Además, se determinó que esta especie presenta una tendencia al agrupamiento de los nidos en el sitio de estudio y esto puede deberse a factores tales como la manera en que esta especie establece sus nuevas colonias transportando los recursos desde la colonia madre, la baja agresividad y las limitaciones en la capacidad máxima de vuelo por tamaño. Estos factores indican que podría existir una baja variabilidad genética en la población estudiada.

AGRADECIMIENTOS. Agradecemos a Colciencias y su programa Jóvenes Investigadores e Innovadores "Virginia Gutiérrez de Pineda, año 2012 y a la Universidad Nacional de Colombia Sede Palmira por ayuda en la financiación del proyecto. A la profesora Guiomar Nates Parra y al laboratorio de abejas de la Universidad Nacional de Colombia LABUN, por su ayuda en la identificación de la especie objeto de estudio. A Kimberly Navarro, Ronald Andrés Viáfara y Juan Sebastián Posada por su disposición y acompañamiento en la localización y referenciación de los nidos.

\section{LITERATURA CITADA}

Araújo, E. D., Costa, M., Chaud-Netto, J. \& Fowler, H.G. (2004). Body size and flight distance in stingless bees (Hymenoptera: Meliponini): inference of flight range and possible ecological implications. Brazilian Journal of Biology. 64, 563-568.

Crane, E., Walker, P. \& Day, R. (1984). Directory of important world honey sources. International Bee Research Association, England. 384 pp.

Fernandez, D.C., Zambrano, G. \& Gonzalez, V. (2010). Comportamiento de nidificación, notas taxonómicas y distribución potencial de Paratrigona eutaeniata (Hymenoptera: Apidae, Meliponini). Revista Colombiana de Entomología. 36, 325-332.

Fierro, M. M., Cruz-López, L., Sánchez, D., Villanueva-Gutiérrez, R. \& Vandame, R. (2012). Effect of Biotic Factors on the Spatial Distribution of Stingless Bees (Hymenoptera: Apidae, Meliponini) in Fragmented Neotropical Habitats. Neotropical Entomology, 41, 95-104.

Free, J. B. (1993). Insect pollination of crops. Second edition. Academic Press. England. 684 pp.

Herrera-Hurtado, S. (2009). Árboles de la Universidad del Valle. Programa editorial Universidad del Valle. Colombia 334 pp.

Hijmans, R. J., Guarino, L. \& Rojas, E. (2001). Computer tools for spatial analysis of plant genetic resources data: 1. DIVA-GIS. Plant Genetic Resources Newsletter, 127, 15-19.

Holdridge, L.R. (1967). Life Zone ecology. Adansonia, 6, 199-203.

Hubbell, S. P. \& Johnson, L. K. (1977). Competition and nest spacing in a tropical stingless bee community. Ecology, 58, 949-963.

Jarau, S. \& Barth, F. G. (2008). Stingless bess of the Golfo Dulce region, Costa Rica (Hymenoptera, Apidae, Apinae, Meliponini). Zugleich Kataloge der oberösterreichischen Landesmuseen, 80, 267-276.
Johnson, L. K. \& Hubbell, S. P. (1974). Aggression and competition among stingless bees: field studies. Ecology, 55, 120-127.

Mantilla, B., Nates-Parra, G. (2006). Distribución y patrones de nidificación de una comunidad de abejas sin aguijón en un agroecosistema subandino (Hymenoptera: Apidae). III encuentro colombiano sobre abejas silvestres. Departamento de Biología, Universidad Nacional de Colombia, Colombia, 60 pp.

Michener, C. D. (1946). Notes on the habits of some Panamanian stingless bee (Hymenoptera, Apidae). Journal of New York Entomological Society, 54, 179-197.

Michener, C. D. (2007). The Bees of the World. Johns Hopkins University Press, United States of America. 953 pp.

Monge, I. (2001) Cómo manejar abejas nativas sin aguijón (Apidae: Meliponinae) en sistemas agroforestales? Agroforestería, 8, 5055.

Nates-Parra, G., Rodriguez, A., Vélez, E. D. (2006). Abejas sin aguijón (Hymenoptera: Apidae: Meliponini) en cementerios de la cordillera oriental de colombia. Acta Biológica Colombiana, 11, 25-35.

Nates-Parra, G., Palacios E. \& Parra A. (2008). Efecto del cambio del paisaje en la estructura de la comunidad de abejas sin aguijón (Hymenoptera: Apidae) en Meta, Colombia. Revista de Biología Tropical, 56, 1295-1308.

Nogueira-Neto, P. (1997). Vida e criação de abelhas indígenas sem ferrão. Editora Nogueirapis. Brasil. 446 pp.

Perry, J. (1995). Spatial Analysis by distance indices. Journal of Animal Ecology, 64: 303-314.

Perry, J. \& Hewitt, M. (1991). A new index of aggregation for animal counts. Biometrics, 47, 1505-1518.

Roubik, D. W. (1979). Nest and colony characteristics of stingless bees from French Guiana (Hymenoptera: Apidae). Journal of the Kansas Entomological Society, 52, 443-470.

Roubik, D. W. (1983). Nest and colony characteristics of stingless bees from Panama (Hymenoptera: Apidae). Journal of the Kansas Entomological Society, 56, 327-355.

Roubik, D. W. (1989). Ecology and natural history of tropical bees. Cambridge University Press, United States of America. 520 pp.

Roubik, D. W. (1992). Stingless bess: a guide to Panamanian and Mesoamerican species and their nests (Hymenoptera: Apidae: Meliponinae). In: D. Quintero \& A. Aiello (Eds.). Insects of Panama and Mesoamerica. Oxford University Press. Oxford. United States of America. 122 pp.

Slaa, E. J. (2003). Foraging Ecology of stingless bees: from individual behavior to community ecology. Universiteit Utrecht. Netherlands. 181 pp.

Slaa, E. J. (2006). Spatial nesting patterns in a Neotropical stingless bee community: do bees compete for food? Proceedings of the Netherlands Entomological Society Meeting, 17, 71-78.

Vásquez, C., Batis, A., Alcocer, M., Gual M. \& Sánchez C. (1999). Árboles y arbustos potencialmente valiosos para la restauración ecológica y la reforestación. Reporte técnico del proyecto JO84. CONABIO - Instituto de Ecología, UNAM, México, 15 pp.

Vergara, C., Villa, A. \& Nates, G. (1986). Nidificación de meliponinos (Hymenoptera: Apidae) de la región central de Colombia. Revista de Biología Tropical. 34, 181-184. 